\title{
Pancreas Transplantation: Solid Organ and Islet
}

\author{
Shruti Mittal, Paul Johnson, and Peter Friend \\ Nuffield Department of Surgical Sciences, University of Oxford, Oxford OX3 9DU, United Kingdom \\ Correspondence: peter.friend@nds.ox.ac.uk
}

Transplantation of the pancreas, either as a solid organ or as isolated islets of Langerhans, is indicated in a small proportion of patients with insulin-dependent diabetes in whom severe complications develop, particularly severe glycemic instability and progressive secondary complications (usually renal failure). The potential to reverse diabetes has to be balanced against the morbidity of long-term immunosuppression. For a patient with renal failure, the treatment of choice is often a simultaneous transplant of the pancreas and kidney (SPK), whereas for a patient with glycemic instability, specifically hypoglycemic unawareness, the choice between a solid organ and an islet transplant has to be individual to the patient. Results of SPK transplantation are comparable to other solid-organ transplants (kidney, liver, heart) and there is evidence of improved quality of life and life expectancy, but the results of solitary pancreas transplantation and islets are inferior with respect to graft survival. There is some evidence of benefit with respect to the progression of secondary diabetic complications in patients with functioning transplants for several years.

Effective medical therapy with exogenous inEsulin has been available for patients with diabetes since 1922, and the majority of patients are well managed on this basis. However, a proportion of patients develop life-threatening complications of diabetes that fail to respond to the most careful insulin regimes. These patients, in whom there is a failure of effective medical therapy, fall broadly into two categories: progression of diabetes renal failure and complications and instability of glycemic control.

Progression of Diabetes-Related Renal Failure and Other Secondary Complications

An unequivocal body of evidence shows that close glycemic control is beneficial to the patient in relation to the onset and progression of sec- ondary complications (nephropathy, neuropathy, and retinopathy) (The Diabetes Control and Complications Trial Research Group 1993). However, tight glycemic control using exogenous insulin is associated with a higher incidence of severe hypoglycemic episodes, making perfect glycemic control unachievable.

\section{Life-Threatening Instability of Glycemic Control}

Some patients with difficult-to-manage diabetes experience hypoglycemic unawareness; not only are these patients liable to hypoglycemic events, but they also have no warning of such an episode and are therefore unable to react in time to prevent hypoglycemic coma. Patients with frequent hypoglycemic unawareness re-

Editors: Laurence A. Turka and Kathryn J. Wood

Additional Perspectives on Transplantation available at www.perspectivesinmedicine.org

Copyright (C) 2014 Cold Spring Harbor Laboratory Press; all rights reserved; doi: 10.1101/cshperspect.a015610

Cite this article as Cold Spring Harb Perspect Med 2014;4:a015610 
quire constant supervision, and many cannot live independently or maintain employment.

It is reasonable to hypothesize that $\beta$-cell transplantation should be the most effective way to maintain physiological glycemic control and also prevent hypoglycemia and hypoglycemic unawareness. However, if transplantation is to be considered the treatment of choice in such patients, then two essential prerequisites must be fulfilled. The first is evidence that transplantation is more effective than other means to maintain stable glycemic control in brittle diabetic patients. The second is evidence that $\beta$-cell transplantation is beneficial with respect to life expectancy, quality of life, or disease progression in patients with renal failure and other secondary complications.

The potential benefit of pancreas transplantation was recognized from the early days of solid organ allografting. Following failed initial attempts at xenotransplantation of fragmented pancreases (Pybus 1924) and the development of immunosuppressive therapies, the first transplanted pancreas was reported by Kelly et al. (1967). The morbidity and mortality associated with these early transplants were extremely high: In a publication in 1970, only two patients out of a series of 10 were alive (Lillehei et al. 1970). In the decades that followed, the morbidity and mortality of solid organ transplantation have decreased greatly, and the rate of success has improved to a level now comparable to kidney and liver transplantation. However, much of the complexity and morbidity of pancreas transplantation still relates to the exocrine component of the gland, and the concept of transplanting only the $2 \%-3 \%$ of cells within the pancreas that produce insulin is clearly attractive. In 1974, Paul Lacy performed the first clinical allotransplant using isolated islets of Langerhans; although initially insulin independent, the recipient subsequently died from sepsis (Karl et al. 1977). It was not until isolation and transplantation of viable islets were first shown to be consistently successful in a report from Edmonton that islet cell transplantation became widespread (Shapiro et al. 2000). In the current era, therefore, there are two therapeutic options for patients with diabetes who require transplantation.

\section{CURRENT PRACTICE AND INDICATIONS FOR TRANSPLANTATION}

\section{Diabetes and Renal Failure}

The large majority of patients undergoing solidorgan pancreas transplantation are those with chronic renal failure secondary to diabetes. These patients, on or approaching the need for dialysis, are candidates for kidney transplantation even in the absence of a pancreas transplant. Kidney transplantation alone for diabetic renal failure has a relatively poor prognosis compared with other indications for this procedure (Cosio et al. 2008; Kuo et al. 2010; Taber et al. 2013); although never subjected to a randomized study, there is increasing evidence that, in patients suitably assessed for the larger procedure, simultaneous pancreas and kidney (SPK) transplantation is associated with improved quality of life (Speight et al. 2010) and life expectancy (van Dellen et al. 2013). The decision as to whether such patients are better advised to undergo kidney transplantation alone, and possible subsequent pancreas after kidney (PAK) transplantation, instead of the combined operation depends on several factors. These include the availability of a potential living donor, the urgency to avoid/come off dialysis, and the likely waiting time for an SPK. There are marked national differences: for example, in the United Kingdom, the majority of patients are listed for an SPK-this reflects the relatively high priority given to such patients in the organ allocation system and the shorter waiting time that results. Currently, SPK and PAK are offered to people with insulin-dependent diabetes (type 1 or type 2) with chronic renal failure. Good results have also been achieved in selected patients following both simultaneous islet kidney (SIK) and islet after kidney (IAK) transplantation (Gerber et al. 2008).

\section{Diabetes and Hypoglycemia}

In patients in whom the primary indication for transplantation is unstable glycemic control, particularly hypoglycemic unawareness, the choice is between solid-organ pancreas transplantation alone (PTA) or islet transplantation. 
This is, essentially, a choice between a much higher-morbidity procedure with a higher likelihood of insulin independence (solid-organ transplantation) versus a much lower-morbidity procedure with a lower chance of long-term function or insulin independence. As is discussed below, insulin independence is less essential if resolution of life-threatening hypoglycemia is the primary indication for transplantation. Indeed, resolution of hypoglycemia unawareness is increasingly seen as the metric of success in islet transplantation rather than insulin independence. This interface between solid-organ and islet transplantation is dynamic, and, with recent improvements in the outcome of islet transplantation in the best centers, the 1-yr and 5-yr graft survival data are much closer between the two modalities. Patients being offered transplantation for hypoglycemic unawareness should be considered for both treatment options. These should be considered as complementary rather than competing treatments, and the decision as to which procedure to perform should be tailored to individual patient needs and preferences.

\section{Diabetes and Other Complications}

The role of PTA in the management of patients who have neither established renal failure (yet) nor hypoglycemic unawareness remains controversial. Offering pancreas transplantation to such a patient is at least partly based on the assumption that benefits will accrue with respect to the progression of secondary diabetic complications and that this benefit will outweigh the complications of long-term immunosuppression. There is evidence (see below) that a successful pancreas transplant may, in the medium term, lead to stabilization or improvement of retinopathy, neuropathy, and cardiovascular disease. However, in a patient who does not require a kidney transplant, this necessitates the patient exchanging the complications of diabetes (metabolic instability, quality of life, life expectancy, secondary complications) with those of the procedure and long-term immunosuppression (operative risk, graft failure, opportunistic infection, cardiovascular disease, can- cer). In the case of a patient with renal failure who would otherwise be receiving a kidney transplant, this balance does not apply because the patient is already committed to the longterm risks of immunosuppression. This is the primary reason that solid-organ pancreas transplantation in the absence of a kidney transplant is much less commonly performed than the combined operation.

This is an area where better evidence is needed; registry analysis is limited by the range and completeness of the recorded data and is unlikely to contribute more than graft and patient survival. Any beneficial effects on secondary complications are likely to require several years to become manifest, necessitating close and repeated measurements of (for example) retinal disease/visual acuity, cardiovascular status, neurological function, and renal function (after PTA). The relatively small numbers of transplants performed by most centers would require this longitudinal study to be performed on a multicenter basis over 5-10 yr.

\section{SECONDARY COMPLICATIONS OF DIABETES}

A crucial question that has not yet been answered definitively is whether successful $\beta$-cell transplantation leads to stabilization or improvement of the secondary complications of diabetes. Many small studies have addressed this issue in both solid-organ and islet transplantation, but the general quality of evidence is relatively poor, and there is a need for larger clinical trials.

Diabetic neuropathy can have a significant impact on the quality of life of patients with diabetes. Autonomic neuropathy causes debilitating gastrointestinal problems that are very difficult to manage, and peripheral neuropathy may cause problems with mobility, pain, or unrecognized injuries that may lead to amputations. Improvements in diabetic neuropathy have been observed with good glycemic control (Callaghan et al. 2012); however, evidence in the context of solid-organ pancreas transplantation is limited. Kennedy et al. (1990) showed improvements in neurological function after 
S. Mittal et al.

24 mo of stable transplant pancreatic function. These improvements were seen in motor, sensory, and autonomic neurology and persisted to 10 yr posttransplant, although improvements in autonomic neuropathy were comparatively modest (Navarro et al. 1990). The Edmonton group were unable to show a statistically significant difference in neuropathy between an islet transplant and the best medical therapy group in a crossover trial (Thompson et al. 2011). One obstacle to research in this area is the lack of a gold standard outcome measure. The recent establishment of confocal corneal microscopy as a reliable, noninvasive, readily repeatable investigation for detecting new nerve growth may address this, and early evidence after pancreas transplantation is promising (Shtein and Callaghan 2013; Tavakoli et al. 2013).

Diabetic retinopathy is the leading cause of blindness in young people, and impairment in vision can severely limit independence and opportunities for employment. Giannarelli et al. (2006) studied 33 Type 1 diabetic patients who underwent PTA, matched with 35 controls and followed for a mean of $30 \mathrm{mo}$, and found significant improvement in the incidence of proliferative retinopathy and the need for laser treatment, and also in nonproliferative retinopathy. However, a high proportion of patients have advanced retinopathy at the time of pancreas transplantation, making stabilization rather than improvement a more realistic goal. Stabilization of disease progression has been observed in several studies (Chow et al. 1999), although the absence of a large comparative study means that it remains unclear whether this is a deviation from the natural progression of advanced retinopathy (Sosna et al. 1998). Data from islet transplantation are similarly sparse; Lee et al. (2005) examined eight patients and found improvement in one and stabilization in seven patients with diabetic retinopathy. Data from the cross-over study performed by the Edmonton group showed significant differences in progression between an islet transplant and best medical therapy (Thompson et al. 2008). However, in order to establish the real effect of transplantation on vision, a large prospective study involving longitudinal retinal images and a relevant control group will be necessary. It has been suggested that rejection episodes may have a deleterious effect on retinopathy, causing sudden deterioration (Scheider et al. 1991). In addition, the increased risk of cataracts following transplantation must also be considered. However, the indications for transplantation would expand significantly if it were proven definitively that transplantation is effective in preventing the progression of diabetic retinopathy.

The effect of pancreas transplantation on renal function is highly relevant to clinical practice but is confounded by the nephrotoxic effect of calcineurin inhibitor drugs, with which most pancreas transplant patients are treated. In patients with already significant diabetic nephropathy (glomerular filtration rates $<40 \mathrm{~mL} / \mathrm{min}$ ), the drug-induced detriment in renal function may advance the need for dialysis. PTA transplantation is therefore contraindicated, but such patients are some years away from dialysis and not yet eligible for a kidney under current allocation systems. There is currently no satisfactory route for the management of this group of patients, but if there were evidence that pancreas transplantation prevents the progression of diabetic renal disease, this may justify much earlier transplantation in such patients. In addition, these patients may benefit from the use of calcineurin-inhibitor-avoiding immunosuppression, although there is little published literature in this area. In a much-cited study, Fioretto et al. (1998) followed eight nonuremic patients who underwent PTA. Although the glomerular filtration rate declined by $5 \mathrm{yr}$ (108 to $74 \mathrm{~mL} / \mathrm{min}$ ), it remained stable at $10 \mathrm{yr}$. Protocol biopsies at 5 and $10 \mathrm{yr}$ showed a decrease in the glomerular and tubular basement membrane thickness at $10 \mathrm{yr}$, with the mesangial fractional volume increased at $5 \mathrm{yr}$ but decreased by $10 \mathrm{yr}$. In islet recipients, the Edmonton group showed a slower decline in GFR after transplantation compared with best medical therapy (Thompson et al. 2011).

Cardiac disease is the leading cause of death in patients with diabetic renal failure. There is evidence that pancreas-kidney transplantation reduces the cardiovascular death rate (La Rocca et al. 2001), and functional studies have shown 
improvement in blood pressure and dyslipidemia compared with kidney transplantation alone (Luan et al. 2007). Benefits have also been shown with respect to systolic and diastolic ventricular function (Fiorina et al. 2000; La Rocca et al. 2001) and endothelial dysfunction (Fiorina et al. 2003; Stadler et al. 2009). However, low cardiac-event rates after transplantation may also reflect stringent cardiac screening and preoperative optimization. Long-term studies linking postoperative functional investigations and clinical end points are needed; current research is addressing cardiac screening techniques and markers of cardiac risk. Fiorina et al. (2003) compared 21 patients with successful islet-kidney transplantation with 13 patients in whom the islet transplant failed, showing benefits with respect to survival and cardiovascular and endothelial function.

\section{BENEFITS OF PANCREAS AND ISLET TRANSPLANTATION}

Although there have been no large-scale randomized controlled trials to establish either whether transplant therapies are more effective than other means of maintaining stable glycemic control, or whether replacement of $\beta$-cell mass is beneficial in patients with secondary complications, ultimately, the object of both solid pancreas and islet transplantation is to enhance quality of life and life expectancy.

For a patient with hypoglycemic unawareness, both quality of life and life expectancy are likely to be improved by resolution of hypoglycemic unawareness, even if they are not rendered insulin independent. Although life-expectancy data are not yet available, emerging quality-oflife data do, indeed, suggest a significant benefit from successful islet transplantation (Bassi and Fiorina 2011) and solid-organ transplantation (Speight et al. 2010).

For patients undergoing transplantation because of secondary diabetic complications ( particularly kidney damage), any benefit is likely to require longer-term function (several years) and insulin independence ( probably). There is considerable evidence (although not from randomized trials) that successful solid-organ trans- plantation increases life expectancy. Tyden et al. (1999) showed that, in a reasonably homogeneous group of patients with diabetic renal failure, those who achieve stable function of a combined pancreas and kidney transplant had substantially improved 10-yr survival compared with the group with stable kidney transplant function alone. Registry analyses confirm the benefit of SPK transplantation versus kidney transplantation alone (and of kidney transplantation versus dialysis). Ojo et al. (2001) showed an almost doubling of life expectancy in patients undergoing the combined operation compared with those who underwent deceased-donor kidney transplantation alone for diabetic renal failure. Clearly, however, these groups were not matched for age or morbidity, although all patients were selected as suitable for transplantation of one or both organs.

\section{SUCCESS OF PANCREAS AND ISLET TRANSPLANTATION}

The outcomes of both solid pancreas and islet transplantation have improved progressively. One- and 5-yr results of SPK transplantation are now close to those of kidney, liver, and heart transplantation (Kandaswamy et al. 2013). Success, however, requires definition. Conventionally, a successful solid-organ pancreas transplant is defined by insulin independence. This is a rational policy based on the assumption that only perfect glycemic control is likely to achieve the benefits in relation to life expectancy and secondary complications. However, as discussed above, in the case of patients with unstable glycemic control undergoing islet transplantation, the primary objective is the resolution of hypoglycemia awareness, and not the restoration of normoglycemia. There is now good evidence that this does not require insulin independence but rather the consistent production of C peptide (Ryan et al. 2004). For this reason, lack of hypoglycemia unawareness is the generally accepted definition of success in islet transplantation.

Current pancreas graft survival in patients undergoing SPK is $85 \%$ at $1 \mathrm{yr}$ and $73 \%$ at $5 \mathrm{yr}$ (Figs. 1-3) ( Kandaswamy et al. 2013). Nearly all 
S. Mittal et al.

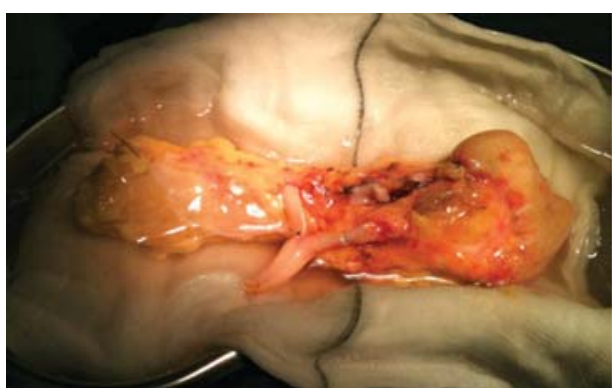

Figure 1. Back-table preparation of pancreas graft for implantation. The segment of donor duodenum is shortened, the donor spleen is removed, and retroperitoneal tissue is removed/ligated. The arterial supply via the superior mesenteric and splenic arteries is attached to a Y graft, usually from the bifurcation of the donor common iliac artery in order to provide a single arterial inflow. Venous outflow via portal vein (shown). (Photograph courtesy of James Gilbert, Oxford Transplant Centre.)

the improvement in graft outcome in recent decades has been achieved during the first $12 \mathrm{mo}$ postoperatively with much less in subsequent years (in line with other solid-organ transplant outcomes). In solid-organ transplantation, SPK outcomes are consistently superior to those from PTA or PAK transplantation (Gruessner et al. 2012). Patients undergoing PTA can expect 1and 5-yr pancreas graft survival of $80 \%$ and $58 \%$, and those undergoing PAK can expect $77 \%$ and $56 \%$ (Kandaswamy et al. 2013). It is generally assumed that the main reason for the discrepancy between SPK and PTA/PAK outcomes is the presence of a kidney from the same donor in the former. This allows renal function to be used as a surrogate biomarker for rejection; not only is it possible to measure renal function in a more sensitive manner, but also the kidney is amenable to biopsy. The greatest risk of graft loss still occurs within the first year posttransplant, and, in particular, within the first 3 mo, when failure is often associated with reperfusion pancreatitis and sepsis due to ischemia-reperfusion injury, a potentially fatal complication. Pancreas transplantation, with or without kidney transplantation, has a patient survival rate of $95 \%$ at $1 \mathrm{yr}$ and $85 \%$ at $5 \mathrm{yr}$.

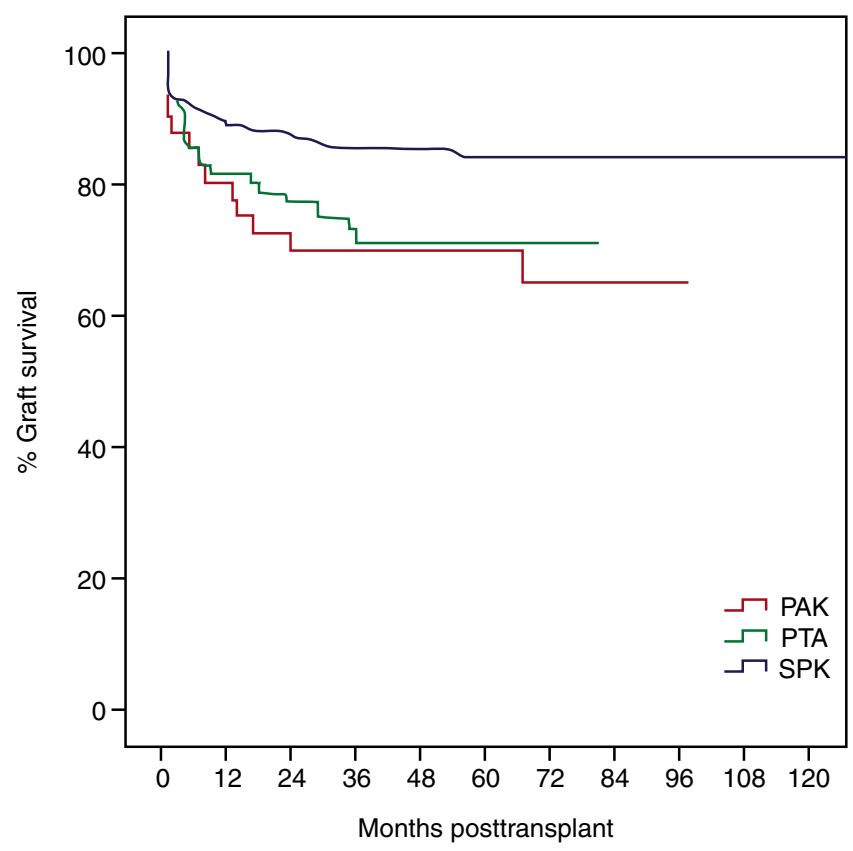

Figure 2. Pancreas graft survival (defined by insulin independence) and patient survival after transplantation at the Oxford Transplant Centre. SPK, Simultaneous pancreas kidney transplant; PTA, pancreas transplant alone; PAK, pancreas after kidney. 


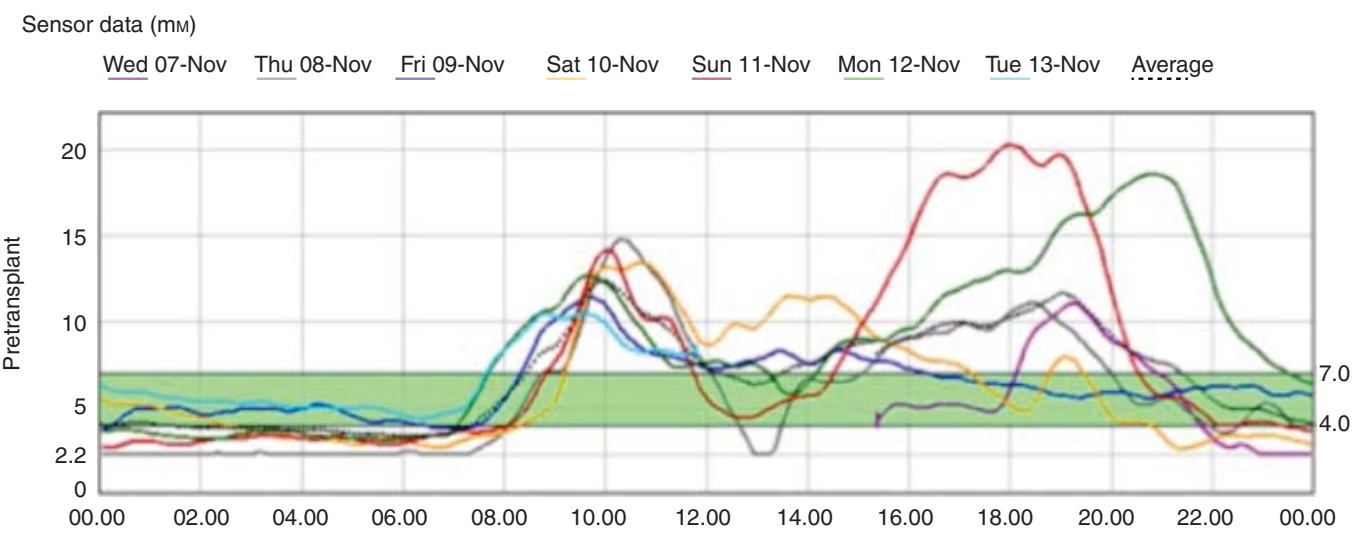

Sensor data (mM)

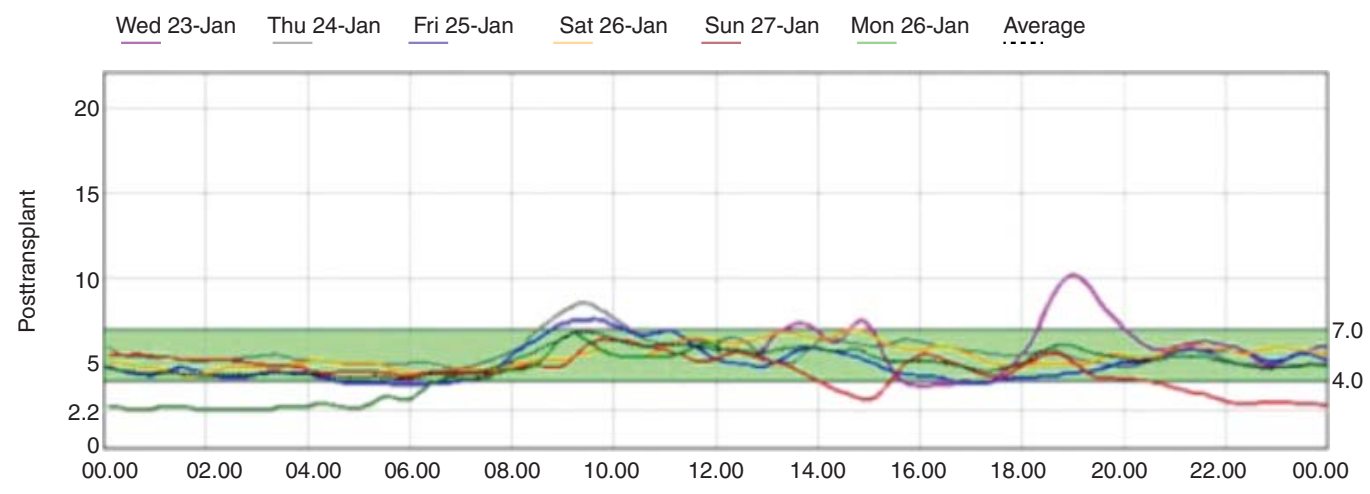

Figure 3. Continuous glucose monitoring before and after islet transplantation performed within the Oxford Islet Transplant Programme, showing improved glycemic control.

The results of islet transplantation have improved greatly over the past decade, in terms both of resolution of hypoglycemic unawareness and achievement of insulin independence. In the 1990s, the Islet Transplant Registry reported an insulin-independence rate of only $11 \%$ in almost 500 islet transplant recipients. However, the introduction of the steroid-free, repeat infusion Edmonton Protocol significantly improved outcomes (Shapiro et al. 2000), which were confirmed by the multicenter Immune Tolerance Network (ITN) trial results of 58\% 1-yr insulin independence in 36 patients (Shapiro et al. 2006). Longer-term results of the original Edmonton Protocol showed a high rate of attrition, with only $\sim 10 \%$ of 65 patients remaining insulin independent $5 \mathrm{yr}$ later (Ryan et al. 2005a), although $\sim 80 \%$ of patients remained C-peptide positive with well-controlled HbAlc. In recent years, the results of islet transplantation have improved. The Islet Transplant Registry reported a 3-yr graft function of only $19 \%$ in 2001, whereas in 2009, the Collaborative Islet Transplant Registry (CITR), with data from islet transplant centers of variable experience, reported that this had risen to $45 \%$.

Islet transplantation has been seen to be even more successful in some centers of excellence. The Minneapolis team achieved 5-yr insulin-independence rates of $50 \%$ in 25 patients using single islet infusions (Ryan et al. 2005a), albeit in recipients with low body-mass indices. The Lille group reported 57\% insulin independence at $3.3 \mathrm{yr}$ posttransplantation in 14 patients (Campbell et al. 2007). The Edmonton team recently presented excellent 5 -yroutcomes using alemtuzumab as the induction agent. Although the longer-term results of islet transplantation 


\section{S. Mittal et al.}

Table 1. $\beta$-Cell transplantation indications, contraindications, risks, and benefits

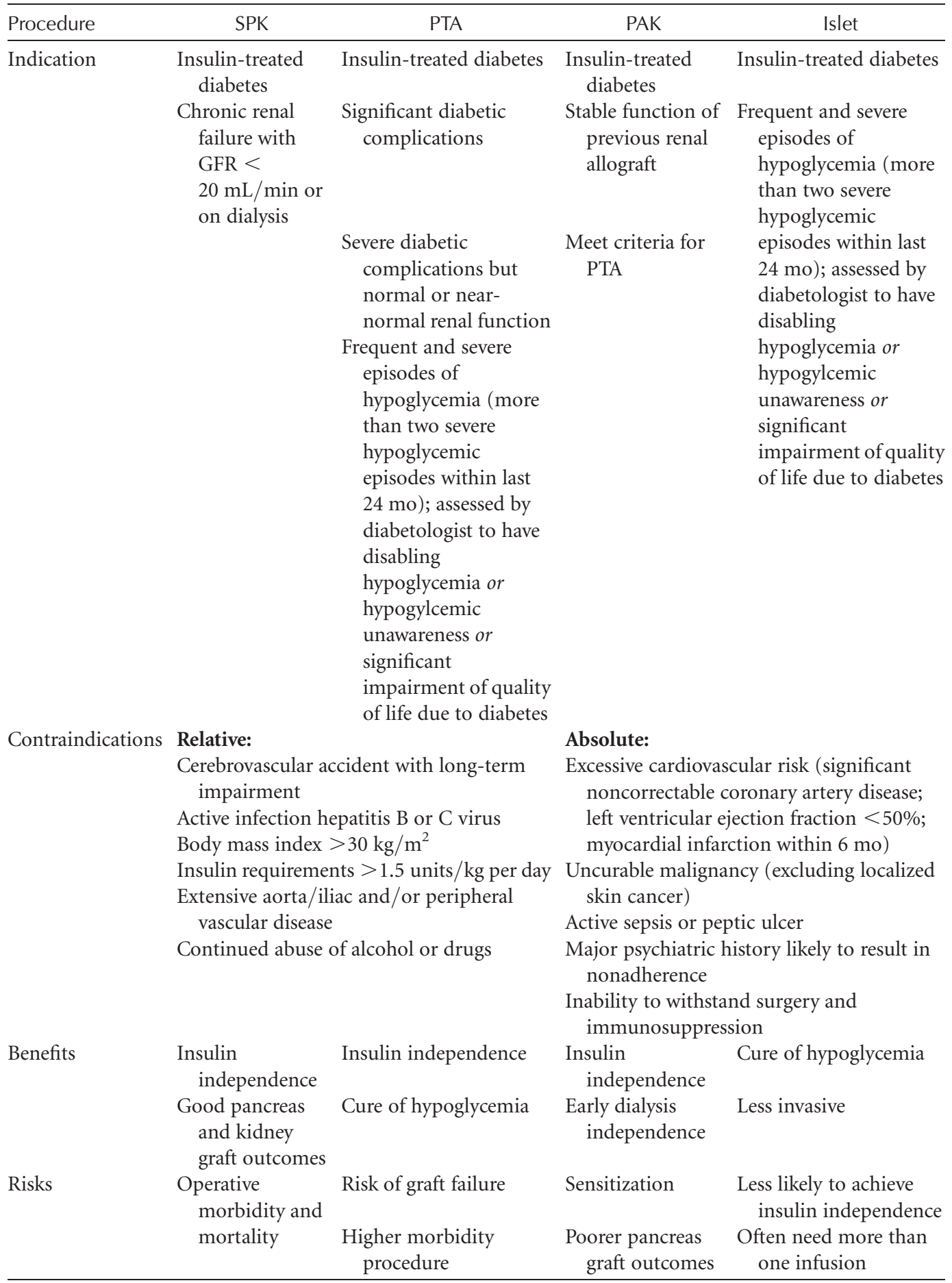

SPK, Simultaneous pancreas kidney transplant; PTA, pancreas transplant alone; PAK, pancreas after kidney. 
are inferior to those of solid-organ transplantation, the much lower morbidity of islet transplantation does enable the possibility of repeat transplantation. However, this carries the risk of sensitizing the recipient to HLA antigens (Campbell et al. 2007; Naziruddin et al. 2012), which may disadvantage any patient who requires kidney transplantation at a subsequent stage.

\section{$\beta$-CELL TRANSPLANTATION-THE PROCESS}

\section{Organ Donation}

Donor selection for pancreas transplantation is more stringent than for other organs, thus limiting the number of potential donors available. Conservative acceptance practices mean that offered pancreases are often declined; indeed, organs may be declined on the basis of donor alcohol use, family history of diabetes, or serum amylase, none of which has been found to be associated with graft outcome (Axelrod et al. 2010). Many donor organs are found to be abnormal at the time of retrieval with fibrosis or fat deposition within the gland. Although steatotic organs often provide good immediate function if transplanted, these organs are liable to severe reperfusion pancreatitis with associated morbidity, and fatty organs are therefore usually discarded. Similarly, donors with a body mass in$\operatorname{dex}(\mathrm{BMI})>30$ are usually declined for solidorgan donation although the evidence base for doing so is limited (Humar et al. 2004; Axelrod et al. 2010). Organ donors up to the age of $60 \mathrm{yr}$ are considered for solid-organ transplantation, but, because donor age has frequently been identified as predictive of graft outcome, pancreases from younger donors are preferred (Proneth et al. 2013). The pancreas is very vulnerable to injury sustained during retrieval, which leads to a higher discard rate of retrieved organs than is seen in other transplant types. For all these reasons, the conversion rate from potential donor to transplant is very low; data from the Scientific Registry of Transplant Recipients show that as little as $20 \%$ of potential donor pancreases are actually retrieved, and of these a further $30 \%$ are discarded after retrieval (Kandaswamy et al. 2013).
Although historically older and higher-BMI donors were preferred for islet transplantation, this was based on isolation rates rather than islet physiology (Lakey et al. 1996; Nano et al. 2005). It is now clear that the optimal donor for islets is younger (Niclauss et al. 2011); this is supported by "in vitro" data of human islets (Lakey et al. 1996; Ihm et al. 2007). Fibrosis and fatty infiltration of the pancreatic parenchyma can prevent pancreas digestion during islet isolation, but fat overlying the pancreas is not in itself a contraindication because this can be removed before digestion.

Cold ischemia is deleterious in both solid organ and islet transplantation, although the recommended limit is shorter $(8 \mathrm{~h})$ in the case of islet transplantation (Lakey et al. 1995; Axelrod et al. 2010). Organs donated after circulatory death (DCD) are used in increasing numbers in solid organ transplantation and have been shown to produce results that are comparable in quality to organs removed from braindead donors (Muthusamy et al. 2012). However, this is achieved by a more selective approach, particularly in terms of donor age and BMI. An objective analysis of donor risk factors has been performed, leading to a donor risk index (Axelrod et al. 2010). The factors associated with the greatest risk (hazard ratio) are as follows: donor age (1.56), DCD status (1.39), black race (1.27), cerebrovascular cause of donor death (1.23), serum creatinine $>2.5 \mathrm{mg} / \mathrm{dL}$ (1.22). Both donor and recipient risk factors need to be incorporated into new organ allocation schemes to optimize the usage of the limited supply of donor pancreases, as well as clinical outcomes for patients undergoing either form of transplantation (Berney and Johnson 2010).

Organ preservation for both solid-organ and islet transplants is performed by static cold storage, most commonly with University of Wisconsin solution, although some units use Celsior or HTK. Despite several trials, no consistent evidence of benefit has been shown, although some studies found a higher incidence of graft pancreatitis, a decreased rate of insulin independence at hospital discharge, and a higher rate of graft loss in HTK-stored pancreases (Stewart et al. 2009). The potential benefits of 
S. Mittal et al.

oxygen carriage using the two-layer technique for islet transplantation (Kawamura et al. 1992) did not to translate from the small animal models to clinical evidence (Kin et al. 2008).

\section{Living Donation and Pancreas Transplantation}

Sutherland et al. (2012) reported 163 livingdonor pancreas transplants using segmental (body and tail of pancreas) organs; these were mainly solid organ, but a small number of islet transplants have been performed. The majority of these were performed at the University of Minnesota; others were performed in countries where deceased donors are rarely available, particularly Japan. There is little evidence of any long-term advantage over deceased-donor transplantation, and subsequent metabolic studies in donors detected significant and concerning metabolic abnormalities (Robertson et al. 2003). This procedure is very rarely performed now.

\section{Solid-Organ Implantation}

The surgical techniques of solid-organ transplantation have changed substantially over the years. The majority of transplant units around the world now transplant the solid pancreas together with a segment of duodenum. The arterial supply via the superior mesenteric and splenic arteries is attached to a Y graft usually from the bifurcation of the donor common iliac artery in order to provide a single arterial inflow. Most units transplant the pancreas with venous drainage to the common iliac vein or inferior vena cava and arterial inflow from the common iliac artery. A much smaller proportion of units advocate venous drainage into the portal venous system. Although associated with more physiological systemic levels of insulin (lower), there is no evidence of substantial benefit with respect to graft or patient survival or other parameters ( $\mathrm{Ba}$ zerbachi et al. 2012).

Drainage of the exocrine excretions of the pancreas has historically been the Achilles heel of pancreas transplantation. Numerous technical modifications have included (1) transplan- tation of the body and tail of the gland (segmental) with occlusion of the pancreatic duct, or drainage into the bladder, the jejunum or the stomach; and (2) transplantation of the whole organ with a "button" of duodenum or duodenal segment, drained into the bladder, jejunum, or duodenum. The expansion of clinical transplantation in the 1990s was based largely on anastomosis of the donor duodenum to the bladder (Sollinger et al. 2009), which had advantages for monitoring (as explained below). In recent years, bladder drainage has been largely supplanted by enteric drainage in which the donor duodenum is anastomosed to the proximal jejunum either directly or via a roux loop. This is clearly a more physiological technique but one that renders the pancreas less easily monitored.

\section{Islet Cell Isolation and Implantation}

The most challenging part of islet transplantation remains the islet isolation. This is a twostage procedure involving pancreas digestion using a combination of enzymatic and mechanical dissociation, and islet purification using density-gradient separation (Ricordi et al. 1989). Although refined in recent decades, this remains a relatively nonspecific, unpredictable method with only $\sim 30 \%$ of pancreases resulting in transplantable yields even in leading centers. Current research efforts are investigating the ultrastructure of the pancreatic exocrine-islet interface in different donor types, so that novel targeted enzyme blends can be developed (Hughes et al. 2006). Islet isolation requires specialized expertise, and, in order to focus skills, many programs are based on a hub-and-spoke model, in which each isolation laboratory provides islets for several implanting centers (Kempf et al. 2005).

Although the original Edmonton Protocol involved the transplantation of "fresh" islets immediately following islet isolation, most centers have now adopted a period of at least $24 \mathrm{~h}$ of islet culture before transplantation (Shapiro et al. 2000). This not only improves the logistics of the transplant, but also enables additional pretransplant islet assessment to be undertaken and 
enables the islets to "recover" following isolation. This is particularly useful for preparations involving larger islets, where central necrosis can be monitored during culture.

Although a few cases of intramuscular islet autotransplants have been reported (Dardenne et al. 2012), the majority of clinical islet allotransplants are performed via the portal vein into the liver sinusoids. This is performed either by a percutaneous, radiologically guided transhepatic approach, or via cannulation of a mesenteric vessel approached laparoscopically or via a mini-laparotomy. Recipients are anticoagulated, and heparin is added to the islet infusion to reduce the immediate inflammatory response, which is believed to be responsible for early islet loss (Cabric et al. 2007). If performed trans-hepatically, embolization of the puncture site in the liver is performed to prevent bleeding (Owen et al. 2003).

\section{Immunosuppression}

Immunosuppression in pancreas transplantation follows a similar pattern to other solidorgan transplants. The majority of units use biological induction (thymoglobulin, alemtuzumab, or basiliximab) followed by a combination of tacrolimus and mycophenolate, and the use of steroids is advocated by some but not all units. There is no conclusive evidence regarding optimal induction therapy; single center studies are small, and of the two multicenter trials, the largest examined an agent (daclizumab) that is no longer available, and the other used variable induction agents (Niederhaus et al. 2013). The use of non-nephrotoxic medication is attractive, but experience in the use of sirolimus following pancreas transplantation remains limited (Kandula et al. 2012).

Similarly, various immunosuppressive regimes have been used for islet transplantation. A feature of the successful Edmonton Protocol was a steroid-free regime, using tacrolimus and sirolimus as maintenance (Shapiro et al. 2000). Since then, many centers have replaced sirolimus with mycophenolate. The use of alemtuzumab-induction therapy is associated with encouraging longer-term function.
In the future, the application of currently experimental methods of immunomodulation are likely to change the practice of pancreas transplantation not only by reducing the morbidity of immunosuppression but also by reducing the adverse effects of unrecognized rejection. Treatment with T-regulatory cells and/ or mesenchymal stem cells may substantially alter the practice of pancreas transplantation.

\section{Graft Monitoring}

Monitoring of both solid-organ and islet transplants is a major challenge. The lack of any simple marker of early graft injury is problematic, with no equivalent to the glomerular filtration rate in kidney transplantation. Hyperglycemia is a relatively late event and often signifies substantial and irreversible graft injury. Glycemic stability is closely monitored and graft function is measured by basal and stimulated C-peptide levels. In islet transplantation, several useful metabolic scores have been introduced to assess graft function objectively. These include the $\beta$ score, a composite of fasting glucose, $\mathrm{HbA}(1 \mathrm{c})$, stimulated C-peptide, and insulin requirement (Ryan et al. 2005b); the HYPO score, which primarily assesses resolution of hypoglycemia; and the liability index (Ryan et al. 2004).

Surrogate measures, including those that relate to the transplanted exocrine tissue, are used in the monitoring of solid organ grafts. Rises in serum amylase and lipase are commonly associated with pancreatitis, but the relevance of changes in the context of graft rejection is uncertain. In combined pancreas-kidney transplantation, kidney graft function (and rejection) is used as a surrogate for changes presumed to be occurring within the pancreas also (although it is recognized that discordant rejection can occur) (Troxell et al. 2010). When the pancreas is transplanted alone, monitoring of pancreas function is problematic. This may explain the discrepancy in outcomes-unrecognized rejection may account for poorer graft survival in this group.

Bladder drainage of exocrine secretions enables amylase production to be used as a measure of transplant function and also allows bi- 
S. Mittal et al.

opsy of the donor duodenum via cystoscopy. Alterations in levels of urinary amylase are associated with impairment in pancreas graft function (Prieto et al. 1987), but this must be balanced against the substantial complications of bladder drainage, both urological (cystitis) and biochemical (bicarbonate loss and dehydration). Percutaneous, radiologically guided pancreatic transplant biopsy of enterically drained transplants is increasingly advocated and (despite earlier concerns) appears to be safe (Klassen et al. 2002). However, this provides the means of diagnosis of rejection but does not solve the problem of monitoring; the challenge of detecting the early manifestations of rejection remains (see review in Margreiter et al. 2013). The development of donor-specific antibodies posttransplant is associated with rejection and graft failure in the pancreas, as in other solidorgan transplants, and monitoring is advocated.

Radiological and radioisotopic methods to measure $\beta$-cell mass and monitor graft function are of experimental interest but not in clinical use. Metabolic tests indicate graft function and reserve but are unwieldy for the purpose of frequent monitoring. Immunological blood monitoring may give advance warning of an immune reaction, but such methods are not yet in routine clinical use.

\section{Morbidity}

Solid-organ and islet transplantation share similar long-term risks with respect to immunosuppression-opportunistic infections, specific drug side effects, and malignancy. Solid-organ pancreas transplantation is associated with much higher procedure-specific morbidity and mortality with many complications requiring reoperation, including hemorrhage, thrombosis, pancreatitis, and sepsis. Venous thrombosis, once a major cause of morbidity (Stockland et al. 2009), has been partially overcome by careful monitoring of coagulation using thromboelastography or other methods-many patients undergoing SPK are relatively hypercoagulable (Muthusamy et al. 2010). Ischemia-reperfusion injury is manifest by reperfusion pancreatitis leading to sepsis and, sometimes, exocrine leak- age. Subsequent complications include mycotic aneurysm formation due to infection of vascular anastomoses - this appears to be a greater risk in pancreas transplantation, possibly because of contamination by enteric organisms at the time of organ retrieval or at the time of enteric anastomosis.

In contrast, islet transplantation is a safe procedure. The two most serious (but rare) procedure-related complications are portal vein thrombosis and bleeding. Although thrombosis of the main portal vein has been reported after islet transplantation using unpurified islets, the risk of this complication is now reduced substantially owing to more rigorous approaches to purification (reducing the volume of cells infused), anticoagulation, and portal pressure monitoring during infusion (Kawahara et al. 2011). However, segmental thrombosis has been reported in up to $5 \%$ of patients (Owen et al. 2003). This risk has been reduced by adding heparin to the islet preparation and by systemic anticoagulation posttransplantation. Hemorrhage from the hepatic puncture site was reported in up to $8 \%$ of cases (Owen et al. 2003), but this complication has been reduced with the introduction of techniques to embolize the catheter tract following islet infusion.

\section{Challenges in $\beta$-Cell Transplantation}

Currently, the morbidity of solid-organ pancreas transplantation restricts pancreas transplantation to relatively younger and fitter patients (very few patients older than $60 \mathrm{yr}$ are accepted for solid-organ pancreas transplantation). Access to islet transplantation is restricted by different parameters, including body mass index and renal function. As the mortality and morbidity of solid pancreas transplantation diminish and the longer-term outcomes of both solidorgan and islet transplantation improve, the appropriate indications for both procedures will expand, particularly with the increasing incidence of diabetes as well as evidence that transplantation is suitable not only for type 1 diabetics but also for selected insulin-dependent patients with type 2 diabetes (nonobese, non-insulin resistant). 
Pancreas Transplantation

There is a clear challenge to improve the utilization of potential donor organs to the level currently achieved in kidney and liver transplantation. This will require new approaches to organ retrieval, preservation, assessment, and the management of ischemia-reperfusion injury.

The process of islet isolation and culture still results in more than $30 \%$ of donor organs not producing a transplantable islet yield, and this is compounded by the fact that many patients still require two infusions to achieve optimal function. In the future, novel enzyme blends will specifically target the pancreatic matrix of the full range of different donor types.

Developments in immunosuppression will reduce the morbidity of transplantation and expand the criteria for transplantation. Tolerance strategies, including T-regulatory cells, will reduce the long-term risks and further shift the risk-benefit threshold of pancreatic transplantation. Novel methods are needed for graft monitoring by immunological or biochemical biomarkers.

It is widely assumed that the development of stem cell therapy will displace allotransplantation in the management of diabetes. Although this is a reasonable long-term vision, the development of insulin-producing cells is not yet close to the point at which clinical implementation is feasible.

\section{REFERENCES}

Axelrod DA, Sung RS, Meyer KH, Wolfe RA, Kaufman DB. 2010. Systematic evaluation of pancreas allograft quality, outcomes and geographic variation in utilization. Am J Transplant 10: 837-845.

Bassi R, Fiorina P. 2011. Impact of islet transplantation on diabetes complications and quality of life. Curr Diab Rep 11: 355-363.

Bazerbachi F, Selzner M, Marquez MA, Norgate A, Aslani N, McGilvray ID, Schiff J, Cattral MS. 2012. Portal venous versus systemic venous drainage of pancreas grafts: Impact on long-term results. Am J Transplant 12: $226-$ 232.

Berney T, Johnson PR. 2010. Donor pancreata: Evolving approaches to organ allocation for whole pancreas versus islet transplantation. Transplantation 90: 238-243.

Cabric S, Sanchez J, Lundgren T, Foss A, Felldin M, Källen R, Salmela K, Tibell A, Tufveson G, Larsson R, et al. 2007 Islet surface heparinization prevents the instant bloodmediated inflammatory reaction in islet transplantation. Diabetes 56: 2008-2015.
Callaghan BC, Little AA, Feldman EL, Hughes RA. 2012. Enhanced glucose control for preventing and treating diabetic neuropathy. Cochrane Database Syst Rev 6: CD007543.

Campbell PM, Senior PA, Salam A, Labranche K, Bigam DL, Kneteman NM, Imes S, Halpin A, Ryan EA, Shapiro AM. 2007. High risk of sensitization after failed islet transplantation. Am J Transplant 7: 2311-2317.

Chow V, Pai R, Chapman J, O'Connell P, Allen R, Mitchell P, Nankivell BJ. 1999. Diabetic retinopathy after combined kidney-pancreas transplantation. Clin Transplant 13: 356-362.

Cosio FG, Hickson LJ, Griffin MD, Stegall MD, Kudva Y. 2008. Patient survival and cardiovascular risk after kidney transplantation: The challenge of diabetes. $\mathrm{Am} \mathrm{J}$ Transplant 8: 593-599.

Dardenne S, Sterkers A, Leroy C, Da Mata L, Zerbib P, Pruvot FR, Pattou F, Truant S. 2012. Laparoscopic spleenpreserving distal pancreatectomy followed by intramuscular autologous islet transplantation for traumatic pancreatic transection in a young adult. JOP 13: 285-288.

Fioretto P, Steffes MW, Sutherland DE, Goetz FC, Mauer M. 1998. Reversal of lesions of diabetic nephropathy after pancreas transplantation. $N$ Engl J Med 339: 69-75.

Fiorina P, La Rocca E, Astorri E, Lucignani G, Rossetti C, Fazio F, Giudici D, di Carlo V, Cristallo M, Pozza G, et al. 2000. Reversal of left ventricular diastolic dysfunction after kidney-pancreas transplantation in type 1 diabetic uremic patients. Diabetes Care 23: 1804-1810.

Fiorina P, Folli F, Bertuzzi F, Maffi P, Finzi G, Venturini M, Socci C, Davalli A, Orsenigo E, Monti L, et al. 2003. Longterm beneficial effect of islet transplantation on diabetic macro-/microangiopathy in type 1 diabetic kidneytransplanted patients. Diabetes Care 26: 1129-1136.

Gerber PA, Pavlicek V, Demartines N, Zuellig R, Pfammatter T, Wuthrich R, et al. 2008. Simultaneous islet-kidney vs pancreas-kidney transplantation in type 1 diabetes mellitus: A 5 year single centre follow-up. Diabetologia 51: $110-119$.

Giannarelli R, Coppelli A, Sartini MS, Del Chiaro M, Vistoli F, Rizzo G, Barsotti M, Del Prato S, Mosca F, Boggi U, et al. 2006. Pancreas transplant alone has beneficial effects on retinopathy in type 1 diabetic patients. Diabetologia 49: 2977-2982.

Gruessner AC, Sutherland DE, Gruessner RW. 2012. Longterm outcome after pancreas transplantation. Curr Opin Organ Transplant 17: 100-105.

Hughes SJ, Clark A, McShane P, Contractor HH, Gray DW, Johnson PR. 2006. Characterisation of collagen VI within the islet-exocrine interface of the human pancreas: Implications for clinical islet isolation? Transplantation 81: 423-426.

Humar A, Ramcharan T, Kandaswamy R, Gruessner RW, Gruessner AG, Sutherland DE. 2004. The impact of donor obesity on outcomes after cadaver pancreas transplants. Am J Transplant 4: 605-610.

Ihm SH, Moon HJ, Kang JG, Park CY, Oh KW, Jeong IK, Oh YS, Park SW. 2007. Effect of aging on insulin secretory function and expression of $\beta$ cell function-related genes of islets. Diabetes Res Clin Pract 77: S150-S154.

Kandaswamy R, Stock PG, Skeans MA, Gustafson SK, Sleeman EF, Wainright JL, Carrico RJ, Ghimire V, Snyder JJ, 
S. Mittal et al.

Israni AK, et al. 2013. OPTN/SRTR 2011 Annual Data Report: Pancreas. Am J Transplant 13: 47-72.

Kandula P, Fridell J, Taber TE, Sharfuddin A, Yaqub MS, Phillips CL, Chen J, Mujtaba M. 2012. Impact of tacrolimus-sirolimus maintenance immunosuppression on proteinuria and kidney function in pancreas transplant alone recipients. Transplantation 94: 940-946.

Karl RC, Scharp DW, Ballinger WF, Lacy PE. 1977. Transplantation of insulin-secreting tissues. Gut 18: 1062 1072.

Kawahara T, Kin T, Kashkoush S, Gala-Lopez B, Bigam DL, Kneteman NM, Koh A, Senior PA, Shapiro AM. 2011 Portal vein thrombosis is a potentially preventable complication in clinical islet transplantation. Am J Transplant 11: 2700-2707.

Kawamura T, Kuroda Y, Saitoh Y. 1992. Long-term preservation of canine pancreas by a new simple cold storage method using perfluorochemical-The two-layer cold storage method (Euro-Collins' solution/perfluorochemical). Kobe J Med Sci 38: 135-145.

Kelly WD, Lillehei RC, Merkel FK, Idezuki Y, Goetz FC. 1967. Allotransplantation of the pancreas and duodenum along with the kidney in diabetic nephropathy. Surgery 61: $827-837$.

Kempf MC, Andres A, Morel P, Benhamou PY, Bayle F, Kessler L, Badet L, Thivolet C, Penfornis A, Renoult E, et al. 2005. Logistics and transplant coordination activity in the GRAGIL Swiss-French multicenter network of islet transplantation. Transplantation 79: 1200-1205.

Kennedy WR, Navarro X, Goetz FC, Sutherland DER, Najarian JS. 1990. Effects of pancreatic transplantation on diabetic neuropathy. $N$ Engl J Med 322: 1031-1037.

Kin T, Senior P, O'Gorman D, Richer B, Salam A, Shapiro AM. 2008. Risk factors for islet loss during culture prior to transplantation. Transpl Int 21: 1029-1035.

Klassen DK, Weir MR, Cangro CB, Bartlett ST, Papadimitriou JC, Drachenberg CB. 2002. Pancreas allograft biopsy: Safety of percutaneous biopsy-Results of a large experience. Transplantation 73: 553-555.

Kuo HT, Sampaio MS, Vincenti F, Bunnapradist S. 2010. Associations of pretransplant diabetes mellitus, new-onset diabetes after transplant, and acute rejection with transplant outcomes: An analysis of the Organ Procurement and Transplant Network/United Network for Organ Sharing (OPTN/UNOS) database. Am J Kidney Dis 56: $1127-1139$.

Lakey JR, Rajotte RV, Warnock GL, Kneteman NM. 1995. Human pancreas preservation prior to islet isolation. Cold ischemic tolerance. Transplantation 59: 689-694.

Lakey JR, Warnock GL, Rajotte RV, Suarez-Alamazor ME, Ao Z, Shapiro AM, Kneteman NM. 1996. Variables in organ donors that affect the recovery of human islets of Langerhans. Transplantation 61: 1047-1053.

La Rocca E, Fiorina P, di Carlo V, Astorri E, Rossetti C, Lucignani G, Fazio F, Guidici D, Cristallo M, Biachi G, et al. 2001. Cardiovascular outcomes after kidney-pancreas and kidney-alone transplantation. Kidney Int 60: 1964-1971.

Lee TC, Barshes NR, O’Mahony CA, Nguyen L, Brunicardi FC, Ricordi C, Alejandro R, Schock AP, Mote A, Goss JA. 2005. The effect of pancreatic islet transplantation on progression of diabetic retinopathy and neuropathy. Transplant Procs 37: 2263-2265.

Lillehei RC, Simmons RL, Najarian JS, Goetz FC. 1970. Pancreatico-duodenal and renal allotransplantation in juvenile onset, insulin dependent, diabetes mellitus with terminal nephropathy. Langenbecks Arch Chir 326: 88-105.

Luan FL, Miles CD, Cibrik DM, Ojo AO. 2007. Impact of simultaneous pancreas and kidney transplantation on cardiovascular risk factors in patients with type 1 diabetes mellitus. Transplantation 84: 541-544.

Margreiter C, Pratschke J, Margreiter R. 2013. Immunological monitoring after pancreas transplantation. Curr Opin Organ Transplant 18: 71-75.

Muthusamy AS, Giangrande PL, Friend PJ. 2010. Pancreas allograft thrombosis. Transplantation 90: 705-707.

Muthusamy AS, Mumford L, Hudson A, Fuggle SV, Friend PJ. 2012. Pancreas transplantation from donors after circulatory death from the United Kingdom. Am J Transplant 12: 2150-2156.

Nano R, Clissi B, Melzi R, Calori G, Maffi P, Antonioli B, Marzorati S, Aldrighetti L, Freschi M, Grochowiecki T, et al. 2005. Islet isolation for allotransplantation: Variables associated with successful islet yield and graft function. Diabetologia 48: 906-912.

Navarro X, Kennedy WR, Loewenson RB, Sutherland DE. 1990. Influence of pancreas transplantation on cardiorespiratory reflexes, nerve conduction, and mortality in diabetes mellitus. Diabetes 39: 802-806.

Naziruddin B, Wease S, Stablein D, Barton FB, Berney T, Rickels MR, Alejandro R. 2012. HLA class I sensitization in islet transplant recipients: Report from the Collaborative Islet Transplant Registry. Cell Transplant 21: 901908.

Niclauss N, Bosco D, Morel P, Demuylder-Mischler S, Brault C, Milliat-Guittard L, Colin C, Parnaud G, Muller YD, Giovannoni L, et al. 2011. Influence of donor age on islet isolation and transplantation outcome. Transplantation 91: 360-366.

Niederhaus SV, Kaufman DB, Odorico JS. 2013. Induction therapy in pancreas transplantation. Transpl Int 26: 704714.

Ojo AO, Meier-Kriesche HU, Hanson JA, Leichtman A, Magee JC, Cibrik D, Wolfe RA, Port FK, Agodoa L, Kaufman DB, et al. 2001. The impact of simultaneous pancreaskidney transplantation on long-term patient survival. Transplantation 71: 82-90.

Owen RJ, Ryan EA, O’Kelly K, Lakey JR, McCarthy MC, Paty BW, Bigan DL, Kneteman NM, Korbutt GS, Rajotte RV, et al. 2003. Percutaneous transhepatic pancreatic islet cell transplantation in type 1 diabetes mellitus: Radiologic aspects. Radiology 229: 165-170.

Prieto M, Sutherland DE, Fernandez-Cruz L, Heil J, Najarian JS. 1987. Experimental and clinical experience with urine amylase monitoring for early diagnosis of rejection in pancreas transplantation. Transplantation 43: 73-79.

Proneth A, Schnitzbauer AA, Zeman F, Foerster JR, Holub I, Arbogast H, Bechstein WO, Becker T, Dietz C, Guba M, et al. 2013. Extended pancreas donor program-The EXPAND study rationale and study protocol. Transplant Res 2: 12. 
Pybus FC. 1924. Notes on suprarenal and pancreatic grafting. Lancet ii: 550-551.

Ricordi C, Lacy PE, Scharp DW. 1989. Automated islet isolation from human pancreas. Diabetes 38: 140-142.

Robertson RP, Sutherland DE, Seaquist ER, Lanz KJ. 2003. Glucagon, catecholamine, and symptom responses to hypoglycemia in living donors of pancreas segments. Diabetes 52: 1689-1694.

Ryan EA, Shandro T, Green K, Paty BW, Senior PA, Bigam D, Shapiro AM, Vantyghem MC. 2004. Assessment of the severity of hypoglycemia and glycemic lability in type 1 diabetic subjects undergoing islet transplantation. Diabetes 53: 955-962.

Ryan EA, Paty BW, Senior PA, Bigam D, Alfadhli E, Kneteman NM, Lakey JR, Shapiro AM. 2005a. Five-year followup after clinical islet transplantation. Diabetes 54: 2060 2069.

Ryan EA, Paty BW, Senior PA, Lakey JR, Bigam D, Shapiro AM. 2005b. $\beta$-Score: An assessment of $\beta$-cell function after islet transplantation. Diabetes Care 28: 343-347.

Scheider A, Meyer-Schwickerath E, Nusser J, Land W, Landgraf R. 1991. Diabetic retinopathy and pancreas transplantation: A 3-year follow-up. Diabetologia 34: S95S99.

Shapiro AM, Lakey JR, Ryan EA, Korbutt GS, Toth E, Warnock GL, Kneteman NM, Rajotte RV. 2000. Islet transplantation in seven patients with type 1 diabetes mellitus using a glucocorticoid-free immunosuppressive regimen. N Engl J Med 343: 230-238.

Shapiro AM, Ricordi C, Hering BJ, Auchincloss H, Lindblad R, Robertson RP, Secchi A, Brendel MD, Berney T, Brennan DC, et al. 2006. International trial of the Edmonton Protocol for islet transplantation. $N$ Engl J Med 355: $1318-1330$.

Shtein RM, Callaghan BC. 2013. Corneal confocal microscopy as a measure of diabetic neuropathy. Diabetes 62 $25-26$.

Sollinger HW, Odorico JS, Becker YT, D’Alessandro AM, Pirsch JD. 2009. One thousand simultaneous pancreas-kidney transplants at a single center with 22-year follow-up. Ann Surg 250: 618-630.

Sosna T, Saudek F, Dominek Z. 1998. Effect of successful combined renal and pancreatic transplantation on diabetic retinopathy. Acta Univ Palacki Olomuc Fac Med 141: 75-77.

Speight J, Reaney MD, Woodcock AJ, Smith RM, Shaw JA. 2010. Patient-reported outcomes following islet cell or pancreas transplantation (alone or after kidney) in Type 1 diabetes: A systematic review. Diabetic Med 27: 812822.

Stadler M, Theuer E, Anderwald C, Hanusch-Enserer U, Auinger M, Bieglmayer C, Quehenberger P, Bischof M, Kästenbauer T, Wolzt M, et al. 2009. Persistent arterial stiffness and endothelial dysfunction following successful pancreas-kidney transplantation in Type 1 diabetes. $D i$ abet Med 26: 1010-1018.

Stewart ZA, Cameron AM, Singer AL, Dagher NN, Montgomery RA, Segev DL. 2009. Histidine-tryptophan-ketoglutarate (HTK) is associated with reduced graft survival in pancreas transplantation. Am J Transplant 9: 217-221.

Stockland AH, Willingham DL, Paz-Fumagalli R, Grewal HP, McKinney JM, Hughes CB, Walser EM. 2009. Pancreas transplant venous thrombosis: Role of endovascular interventions for graft salvage. Cardiovasc Intervent $R a$ diol 32: 279-283.

Sutherland DE, Radosevich D, Gruessner R, Gruessner A, Kandaswamy R. 2012. Pushing the envelope: Living donor pancreas transplantation. Curr Opin Organ Transplant 17: 106-115.

Taber DJ, Meadows HB, Pilch NA, Chavin KD, Baliga PK, Egede LE. 2013. Pre-existing diabetes significantly increases the risk of graft failure and mortality following renal transplantation. Clin Transplant 27: 274-282.

Tavakoli M, Mitu-Pretorian M, Petropoulos IN, Fadavi H, Asghar O, Alam U, Ponirakis G, Jeziorska M, Marshall A, Efron N, et al. 2013. Corneal confocal microscopy detects early nerve regeneration in diabetic neuropathy after simultaneous pancreas and kidney transplantation. Diabetes 62: $254-260$.

The Diabetes Control and Complications Trial Research Group. 1993. The effect of intensive treatment of diabetes on the development and progression of long-term complications in insulin-dependent diabetes mellitus. N Engl J Med 329: 977-986.

Thompson DM, Begg IS, Harris C, Ao Z, Fung MA, Meloche RM, Keown P, Meneilly GS, Shapiro RJ, Ho S, et al. 2008. Reduced progression of diabetic retinopathy after islet cell transplantation compared with intensive medical therapy. Transplantation 85: 1400-1405.

Thompson DM, Meloche M, Ao Z, Paty B, Keown P, Shapiro RJ, Ho S, Worsley D, Fung M, Meneilly G, et al. 2011 Reduced progression of diabetic microvascular complications with islet cell transplantation compared with intensive medical therapy. Transplantation 91: 373-378.

Troxell ML, Koslin DB, Norman D, Rayhill S, Mittalhenkle A. 2010. Pancreas allograft rejection: Analysis of concurrent renal allograft biopsies and posttherapy follow-up biopsies. Transplantation 90: 75-84.

Tyden G, Bolinder J, Solders G, Brattstrom C, Tibell A, Groth CG. 1999. Improved survival in patients with insulin-dependent diabetes mellitus and end-stage diabetic nephropathy 10 years after combined pancreas and kidney transplantation. Transplantation 67: 645-648.

van Dellen D, Worthington J, Mitu-Pretorian OM, Ghazanfar A, Forgacs B, Pararajasingam R, Campbell B, Parrott NR, Augustine T, Tavakoli A. 2013. Mortality in diabetes: Pancreas transplantation is associated with significant survival benefit. Nephrol Dial Transplant 28: 1315-1322. 


\section{$\&_{\mathrm{CSH}}^{\infty} \&$ Cold Spring Harbor

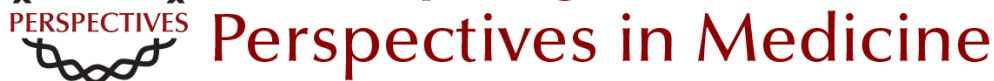

\section{Pancreas Transplantation: Solid Organ and Islet}

Shruti Mittal, Paul Johnson and Peter Friend

Cold Spring Harb Perspect Med 2014; doi: 10.1101/cshperspect.a015610 originally published online March 10, 2014

\section{Subject Collection Transplantation}

Heart Transplantation: Challenges Facing the Field

Makoto Tonsho, Sebastian Michel, Zain Ahmed, et al.

Bioethics of Organ Transplantation Arthur Caplan

Overview of Clinical Lung Transplantation Jonathan C. Yeung and Shaf Keshavjee

Immunological Challenges and Therapies in

Xenotransplantation Marta Vadori and Emanuele Cozzi

Clinical Aspects: Focusing on Key Unique

Organ-Specific Issues of Renal Transplantation Sindhu Chandran and Flavio Vincenti

T-Cell Costimulatory Blockade in Organ

Transplantation

Jonathan S. Maltzman and Laurence A. Turka

Regulatory T-Cell Therapy in Transplantation:

Moving to the Clinic

Qizhi Tang and Jeffrey A. Bluestone

Opportunistic Infections--Coming to the Limits of Immunosuppression?

Jay A. Fishman
Overview of the Indications and Contraindications for Liver Transplantation

Stefan Farkas, Christina Hackl and Hans Jürgen Schlitt

Facial and Hand Allotransplantation Bohdan Pomahac, Ryan M. Gobble and Stefan Schneeberger

Induction of Tolerance through Mixed Chimerism David H. Sachs, Tatsuo Kawai and Megan Sykes

Pancreas Transplantation: Solid Organ and Islet Shruti Mittal, Paul Johnson and Peter Friend

Tolerance--Is It Worth It?

Erik B. Finger, Terry B. Strom and Arthur J. Matas

Lessons and Limits of Mouse Models Anita S. Chong, Maria-Luisa Alegre, Michelle L. Miller, et al.

Effector Mechanisms of Rejection Aurélie Moreau, Emilie Varey, Ignacio Anegon, et al.

The Innate Immune System and Transplantation Conrad A. Farrar, Jerzy W. Kupiec-Weglinski and Steven H. Sacks

For additional articles in this collection, see http://perspectivesinmedicine.cshlp.org/cgi/collection/ 